\title{
The knee at cosmic ray spectra is explained due to interactions at the sources
}

\author{
WANG Bo \& YUAN Qiang
}

In the paper the authors from the Key Laboratory of Particle Astrophysics, Institute of High Energy Physics, Chinese Academy of Sciences in Beijing proposed a new model trying to explain the knee at cosmic ray spectra. The knee kept as a puzzle in cosmic ray physics for nearly half a century. The work was inspired by the recent observation of anomalous excess of electrons and positrons in cosmic rays. The work tries to explain the knee and the electron/positron excess in a single model.

The study is reported in Volume 53, Number 5 of SCIENCE CHINA Physics, Mechanics \& Astronomy because of its significant research value. The work was finished by Dr. Wang Bo and his collaborators from a group of physicists who study cosmic ray physics based on the Yabajing cosmic ray observatory.

Cosmic ray was discovered by Victor Hess in 1912. Yet about hundred years later, the origin of the cosmic rays remains a puzzle today. It is generally believed that the Galactic cosmic rays are accelerated in shock waves at supernovae remnants, however, no convincing evidence has been detected.

The energy spectrum of cosmic rays (CRs) follows a power law with index about -3 in a wide energy range (dN/d $E \sim E^{s}$ ). The "knee" refers to the change of the power law index s from -2.7 to -3.1 at energy $\sim 4 \mathrm{PeV}$, which makes the spectrum seem like the knee of one's leg.

Since the discovery made by Kulikov \& Kristiansen in 1958, how the knee is formed has become to be another classic problem in CR physics. The explanations of the knee include the acceleration limit of Galactic CR sources, the leakage of CRs from the Galaxy, and interactions with matter, photons, neutrinos and so on. However, those models tend to predict a too fast drop of the CR spectra above the knee energy. An additional contribution to the cosmic rays spectra is requested to fit measurements, which is called the "B component" of cosmic rays.

Recently the measurements of the CR spectra around the knee region become more precise, which can even reveal some fine structures of the knee. Especially the result from the Yangbajing observatory shows a sharp break at the knee. Some other experiments have also reported their new measurements with the similar behavior, such as by KASCADE, Aragats-GAMMA, Yakutsk, and MAKET-ANI. Besides the sharp transition at the knee, analysis by Erlykin and Wolfendale reveals several small bumps on the CR spectra near the knee, the so-called fine structures.

Inspired by the result of the anomalous cosmic positron and electron excesses observed by ATIC, PAMELA, HESS and Fermi, the authors proposed a new model trying to solve the knee problem and account for the electron observations simultaneously. The model assumes that the nuclei-photon interaction with electron-positron pair production $\left(\mathrm{p} \gamma \rightarrow \mathrm{pe}^{+} \mathrm{e}^{-}\right)$at the acceleration sources is the origin of the $\mathrm{CR}$ knee. In some CR acceleration sources the photon background is very dense. The high energy charged CR particles will interact with the background inevitably. For the visible light with energy of about $1 \mathrm{eV}$, the reaction for the electron-positron pair produced can only take place when the CRs energy reaches PeV, which is just the energy of the knee. When the interaction takes place, the high energy CR particles will loss a part of its energy and lead the CR spectra fall down more rapidly. Therefore two different power law indices are present in the CR spectra before and after the energy where the pair production takes place. This behavior just accounts for the knee of the CR spectra. At the same time the produced electron-positron pairs can also explain the electron/positron excesses by recent measures.

According to a detailed simulation of the collision process the analysis in this work further shows that the model can well explain the sharp knee due to rapid energy loss once the reaction channel is opened. More interestingly the fine structures near the knee are also reproduced in this interaction model since different CR components have different threshold energies for pair production. Finally the so-called "component B" of Galactic CRs is also explained since the reaction of pair production becomes weak for much higher energy than the threshold energy.

It is proposed in the work that the intense nuclei-photon interaction can be achieved in young supernova remnants. The present work is a new attempt to explain two long-term puzzles, the origin of cosmic rays and the knee of the spectra. The relation between the knee and the recent observation of the electron-positron excess is another interesting feature of the model.

The authors are affiliated at Key Laboratory of Particle Astrophysics, Institute of High Energy Physics, Chinese Academy of Sciences in Beijing and Department of Physics, Shandong University in Jinan. The authors are conducting research mainly in high energy cosmic ray fields.

Funding from the Ministry of Science and Technology of China, the National Natural Sciences Foundation of China (Grant Nos. 10725524 and 10773011), and the Chinese Academy of Sciences (Grant Nos. KJCX2-YW-N13, KJCX3-SYW-N2 and GJHZ1004).

See the articles: Wang B, Yuan Q, Fan C, et al. A study on the sharp knee and fine structures of cosmic ray spectra. Sci China Phys, Mech Astron, 2010, 53(5): 842-847; http://www.springerlink.com/content/0u3k326174317727/Hu H B, Yuan Q, Wang B, et al. On the e+e excesses and the knee of the Cosmic Ray Spectra-Hints of cosmic ray acceleration in young supernova remnants. Astrophys J, 2009, 700: L170-L173; http://iopscience.iop.org/1538-4357/700/2/L170/ 